\title{
Sulla stima di macro, micro e nano-durezza di materiali metallici mediante analisi elasto-plastiche agli elementi finiti
}

\author{
Estimating macro-, micro-, and nano-hardness of metallic \\ materials from elasto-plastic finite element results
}

\author{
Luca Susmel \\ The University of Sheffield, Department of Civil and Structural Engineering, Sheffield, S1 3JD, UK \\ David Taylor \\ Trinity College, Department of Mechanical Engineering, Dublin 2, Ireland
}

\begin{abstract}
RIASSUNTO. Il lavoro sintetizzato nel presente articolo si pone come obiettivo primario quello di investigare la possibilità di stimare, mediante un approccio elasto-plastico agli elementi finiti, la durezza dei materiali metallici convenzionali, e questo sia a livello macroscopico, che a livello microscopico, che, infine, a livello nanoscopico. Per verificare validità e accuratezza della metodologia FEM sviluppata, sono state condotte una serie di analisi sperimentali su tre materiali metallici aventi caratteristiche metallurgiche estremamente diverse: una lega d'alluminio (Al 7075-T6), un acciaio a basso tenore di carbonio (BS970-En3B) e, infine, un acciaio austenitico (AISI 316L). L'indentazione Vickers è stata simulata con analisi elasto-plastiche agli elementi finiti considerando carichi di prova nell'intervallo tra $490 \mathrm{~N}$ e $490 \mu \mathrm{N}$ e calibrando le simulazioni numeriche mediante curve monotone tensione-deformazione ottenute da prove di trazione eseguite utilizzando provini sia di dimensione convenzionale che aventi larghezza della zona calibrata dell'ordine dei $100 \mu \mathrm{m}$.

La sistematica comparazione tra risultati sperimentali e simulazioni numeriche ha posto in evidenza come l'aumentare del valore della durezza misurata al diminuire della dimensione dell'impronta possa essere imputata al ruolo giocato dalla reale morfologia del materiale, ruolo che diventa predominante sulla plasticità convenzionale quando le dimensioni della superficie indentata diventano comparabili con le dimensioni medie della grana cristallina delle leghe esaminate. Tali fenomeni, pertanto, non hanno consentito di estendere l'utilizzo della meccanica del continuo fino ad un livello nanoscopico per determinare correttamente i valori della durezza. Alla luce di questi risultati è stata, però, proposta una semplice metodologia di correzione delle stime eseguite mediante gli elementi finiti che si è dimostrata un valido strumento da utilizzarsi in situazioni di interesse pratico per stimare la durezza dei materiali metallici, indipendentemente dalla dimensione della superficie indentata.
\end{abstract}

ABSTRACT. This paper summarises an attempt of estimating macro-, micro-, and nano-hardness of metallic materials through conventional elasto-plastic finite element (FE) analyses. In more detail, to verify if the classical FE method can successfully be used for such a purpose, initially a series of hardness testes were carried out on three metallic materials characterised by a different elasto-plastic behaviour, i.e., aluminum alloy 7075T6, low-carbon steel BS970-En3B, and, finally, austenitic steel AISI 316L. Subsequently, by making the indentation force vary in the range $490 \mathrm{~N}-490 \mu \mathrm{N}$, Vickers hardness was estimated from elasto-plastic FE models done by using, to calibrate the mechanical properties of the investigated metals, the corresponding 
monotonic stress-strain curves experimentally determined by testing samples having both conventional size and, for austenitic steel AISI 316L, gauge length size equal to approximately $100 \mu \mathrm{m}$.

The systematic comparison between experimental results and numerical simulations suggests that the increasing of the measured hardness value with the decreasing of the indenter size may directly be ascribed to the role played by the actual morphology of the material being tested. In particular, it is seen that conventional elastoplastic continuum mechanics is no longer adequate to estimate metallic material hardness as the size of the indented surface approaches the average size of the grains. Finally, in order to overcome the above limitation by allowing the classical elasto-plastic FE approach to be used also to estimate nano-hardness, a simple engineering method is proposed and subsequently validated through the generated experimental results.

KEYwORDS. Durezza; Nanoindentazione; Elementi finiti; Plasticità.

\section{INTRODUZIONE}

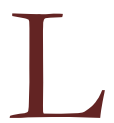

e prove di durezza con penetratore sono da più di un secolo ampiamente impiegate nella pratica industriale per misurare in modo rapido ed economico alcune proprietà meccaniche dei materiali (come, ad esempio, per la stima diretta della tensione di snervamento), nonché per valutare le caratteristiche di trattamenti e lavorazioni superficiali. Queste metodologie di misura, basate sull'uso di penetratori di geometria standard, permettono l'ottenimento di impronte permanenti (aventi la particolarità di essere geometricamente simili al variare della profondità di penetrazione, ovvero, al variare del carico di prova) la cui estensione superficiale consente il computo diretto del valore di durezza, intesa come pressione media esercitata dal penetratore sull'area dell'impronta, area misurata dopo aver rimosso il carico [1-3].

Il forte sviluppo della microelettronica a cui si è assistito negli ultimi decenni (circuiti integrati, microprocessori, ecc.), nonché delle più moderne nanotecnologie (con importanti applicazioni nel campo biomedico), ha di recente portato gli specialisti del settore a rivalutare ulteriormente l'importanza delle prove di durezza basate sull'uso di penetratori. In particolare, viste le ridotte dimensioni di tali componenti, le prove di durezza si sono dimostrate forse le più adatte a rilevare in modo non-distruttivo le proprietà meccaniche dei materiali utilizzati in queste particolari applicazioni. Ovviamente, viste le dimensioni assolute dei componenti di cui doveva essere eseguita la misura della durezza, i carichi di prova sono stati ridotti fino ad ottenere profondità di penetrazione compatibili con le dimensioni del componente da testare.

Uno degli aspetti più interessanti di questo processo di miniaturizzazione delle prove di durezza è stata la progressiva riduzione delle dimensioni assolute dell'impronta, mantenendone, però, inalterata la geometria, con l'evidente vantaggio di poter ottenere misure su diverse scale dimensionali che, almeno dal punto di vista geometrico, potessero essere confrontate in modo diretto. Considerando, invece, in modo più generale il problema della dipendenza del valore della durezza dalla dimensione dell'area indentata, è possibile affermare che quando la profondità dell'impronta, ottenuta mediante penetratori conici o piramidali, supera una certo valore di soglia (che varia al variare del materiale), la durezza misurata risulta praticamente indipendente dal valore del carico applicato: questa è la tipica situazione che si osserva nel caso di misure di macro-durezza su materiali metallici. Al contrario, quando, invece, la profondità di penetrazione è inferiore al valore di soglia a cui si faceva riferimento poco sopra, le misure di durezza diventano fortemente dipendenti dalle dimensioni dell'impronta stessa, tanto che il valore della misura può risultare anche due o tre volte più alto del valore ottenuto mediante un macro-indentatore (questo fenomeno diventa particolarmente evidente quando si eseguono misure su monocristalli o su materiali policristallini, come chiaramente evidenziato dalla diagramma di Fig. 1).

Il lavoro sintetizzato nel presente articolo si incentra sullo studio delle problematiche legate alla stima della durezza di materiali metallici, quando la durezza viene determinata, su diverse scale dimensionali, mediante l'uso di penetratori di tipo Vickers. Interessante è subito osservare che quando ci si riferisce a questa particolare geometria di penetratore, come brevemente accennato poco sopra, sembra essere convinzione comune [5] che il valore della durezza, calcolato secondo la formula:

$$
\mathrm{HV}=\frac{2 \mathrm{p} \cdot \sin 68^{\circ}}{\mathrm{d}^{2}}
$$


sia indipendente dal carico di prova (dove nella precedente equazione $\mathrm{p}$ è la forza applicata e d la dimensione dell'impronta). Tale convinzione, ampiamente supportata da sistematiche campagne sperimentali, risulta però valida fintanto che le dimensioni dell'impronta sono sufficientemente grandi rispetto alla grana cristallina, ovvero, tale affermazione è valida solo per prove di macro-durezza. Al contrario, alcuni studi $[6,7]$ hanno evidenziato come esista una forte dipendenza del valore della durezza Vickers dalla dimensione dell'impronta quando questa diventa comparabile con la dimensione della grana cristallina. In più, tali studi hanno posto in evidenza come un tale effetto scala non possa essere spiegato invocando unicamente la tradizionale teoria della plasticità, a meno che questa non venga pesantemente modificata onde estenderne la validità anche su scala microscopica. In particolare, si può osservare come l'aumento apparente di durezza a cui si assiste, per uno stesso materiale, passando dal campo macroscopico al campo microscopico, non sembra essere spiegabile solo con considerazioni puramente dimensionali (ovvero, ricorrendo al classico effetto scala): secondo le più moderne interpretazioni, un tale incremento apparente sembra sia legato alla proliferazione di dislocazioni geometricamente necessarie, proliferazione indotta dai gradienti di deformazione che vengono imposti al materiale durante il processo di indentazione [8-10]. Più precisamente, deformazioni non uniformi dell'ordine di grandezza del $\mu \mathrm{m}$ producono una densità di dislocazioni geometricamente necessarie che risulta essere comparabile, o a volte anche superiore, alla densità di dislocazioni statisticamente presenti. In altre parole, maggiore è l'ordine di grandezza della deformazione in presenza di forti gradienti, più alta è la densità relativa di dislocazioni geometricamente necessarie e più alto è il loro contributo all'incrudimento: queste rappresentano le basi sui cui è stata recentemente sviluppata la teoria della plasticità fondata sul gradiente della deformazione (strain gradient plasticity) [10].

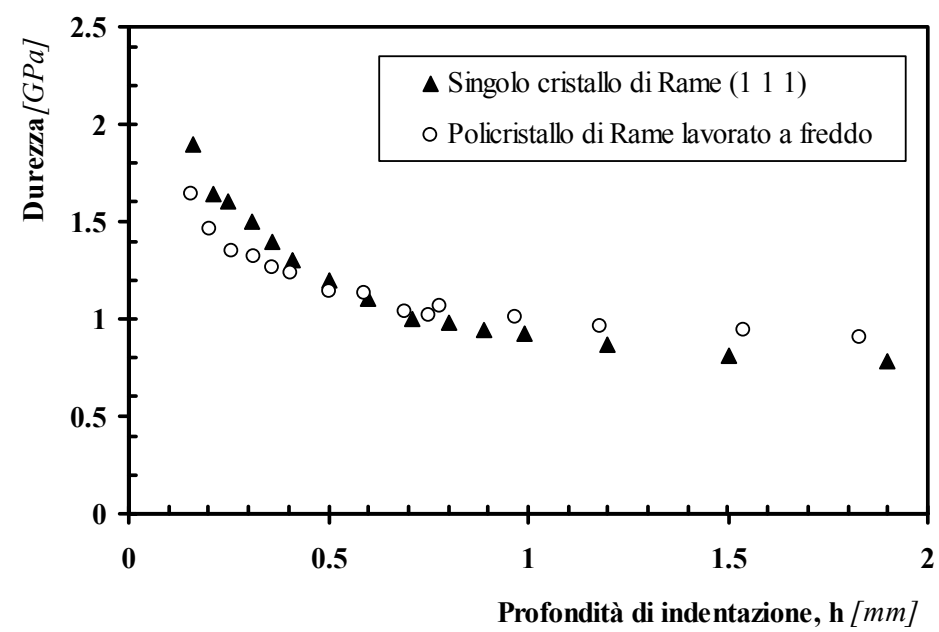

Figura 1: Dipendenza della durezza, misurata mediante una punta Berkovich, dalla profondità di indentazione di un singolo cristallo di rame e di un agglomerato policristallino di rame lavorato a freddo [4].

Figure 1: Relationship between Berkovich hardness and indentation depth in a single crystal as well as in a cold-rolled polycrystal of copper [4].

Partendo dalle problematiche brevemente menzionate poco sopra, il presente lavoro riporta i risultati ottenuti nella previsione, mediante analisi elasto-plastiche agli elementi finiti, di macro-, micro- e nano-durezza di tre materiali metallici comunemente utilizzati nella realizzazione di componenti meccanici. In particolare, il presente lavoro si pone come obiettivo primario quello di formalizzare una possibile correlazione che leghi misure di durezza ottenute su diverse scale dimensionali mediante l'uso di simulazioni agli elementi finiti e assumendo che il materiale obbedisca ancora alle leggi della meccanica del continuo. La semplice procedura proposta si basa sull'uso di un valore di durezza utilizzato per la calibrazione del modello stesso e sulla curva tensione-deformazione del materiale, curva determinata in campo elastoplastico a mezzo di prove convenzionali di trazione e descrivibile mediante la nota relazione di Ramberg-Osgood [11].

Interessante è osservare che il metodo proposto non solo ha consentito di legare in modo semplice $i$ valori di durezza determinati su scala macro, micro e nano, ma ha permesso anche di individuare il limite di applicabilità della teoria della plasticità convenzionale nella modellazione delle misure di durezza superficiale, suggerendo, allo stesso tempo, che il fenomeno della strain gradient plasticity possa essere tenuto, in un certo qual modo, in considerazione mediante un parametro direttamente legato al comportamento plastico macroscopico del materiale.

Per concludere, e prima di entrare nel dettaglio della metodologia proposta, è importante sottolineare che, onde restare all'interno del campo di validità della meccanica del continuo, quanto formalizzato nel presente lavoro si basa sulla 
assunzione che il materiale sia omogeneo, continuo e isotropo: la dimensione del grano e gli effetti di anisotropia locale dei cristalli non vengono, pertanto, tenuti in alcuna considerazione.

\section{MOdELlazione DELla DEFORMAZIONE PLASTICA DOVUTA ALLA INDENTAZIONE MEDIANTE ANALISI NUMERICHE AGLI ELEMENTI FINITI}

$\mathrm{P}$ rendendo spunto da quanto già fatto, sia in termini sperimentali che in termini di modellazione, da Giannakopoulos et al. [12], è stata messa a punto una procedura adatta a modellare agli elementi finiti il problema dell'indentazione mediante penetratore Vickers. Tale procedura è stata basata sull'utilizzo, per la determinazione della soluzione numerica, del codice di calcolo commerciale ANSYS ${ }^{\circledR}$.

Inizialmente, e per quanto riguarda la parte di modellazione solida, sfruttando le proprietà di simmetria rispetto a otto piani del penetratore tipo Vickers, la geometria del problema in esame è stata fortemente semplificata secondo quanto riportato in Fig. 2. Si è poi ottenuta una ulteriore semplificazione, considerando il penetratore come un corpo perfettamente rigido e indeformabile (e questo in virtù della elevata rigidezza del diamante comparata a quella dei metalli di cui si doveva eseguire la stima della durezza).

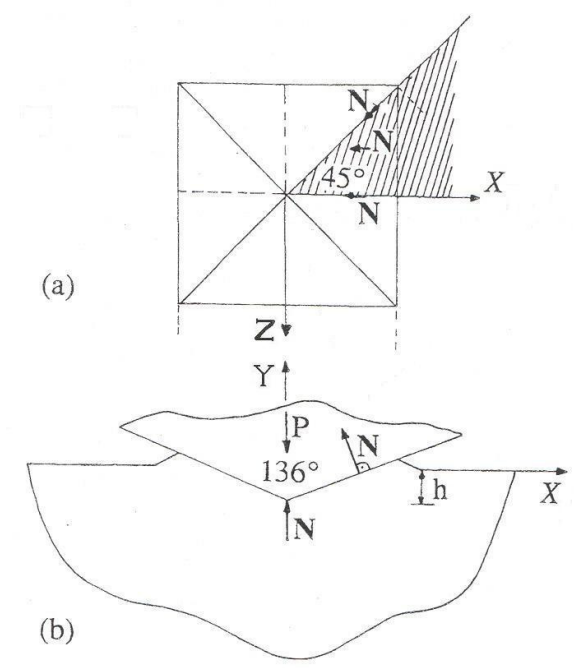

Figura 2: Rappresentazione schematica di un test di durezza eseguito utilizzando un penetratore di tipo Vickers.

Figure 2: Schematic sketch of a Vickers test.

Le analisi condotte sono state trattate come problemi isotermi e con applicazione quasi-statica del carico: queste assunzioni hanno permesso di escludere gli effetti dinamici dovuti all'energia cinetica del penetratore.

La mesh del solido di cui doveva essere modellata l'impronta generata dal penetratore Vickers è stata creata in modo che in corrispondenza, e nei dintorni, dell'area di contatto la dimensione degli elementi fosse piuttosto contenuta, onde determinare correttamente la distribuzione del campo elasto-plastico di tensione. Per valutare la bontà della mesh realizzata è stata condotta una accurata e sistematica analisi di convergenza che ha mostrato come il numero di elementi in contatto in condizioni di massimo carico doveva essere almeno pari ad otto in ogni direzione. Più lontano dalla zona di contatto, invece, la densità della mesh è stata gradatamente ridotta, visto che tali elementi risultavano essere evidentemente caratterizzati da un valore della deformazione decisamente inferiore. La Fig. 3a e, nel dettaglio, la Fig. 3b mostrano la distribuzione della mesh utilizzata per condurre le analisi elastoplastiche: il solido su cui doveva essere modellata l'impronta dell'indentatore è stato meshato utilizzando un totale di 22261 nodi e 5219 elementi brick del tipo "Solid 186" [13]; inoltre attraverso il "Contact Wizard" di ANSYS ${ }^{\circledR}$ è stato creato l'accoppiamento di contatto tra il penetratore e il solido da testare, con l'aggiunta di elementi "Target 170" e "Contact 174" [13].

Per garantire poi un adeguato livello di precisione nella soluzione, precisione che dipendeva dal numero di elementi in contatto sull'area dell'indentazione, mantenendo contemporaneamente i tempi di calcolo a livelli accettabili, lo studio di convergenza ha evidenziato la necessità di scalare, secondo una certa proporzione, le dimensioni sia del provino che dell'impronta generata in funzione del carico di prova. Una tale necessità si è in pratica tradotta nel determinare la 
soluzione di modelli geometricamente simili e aventi lo stesso numero di nodi, secondo quanto descritto nel grafico di Fig. 4.

Per quanto riguarda i vincoli applicati, al solido indentato sono state applicate condizioni di simmetria sulle facce laterali, bloccando anche gli spostamenti verticali della sua base. Il carico al penetratore, avente direzione coincidente con l'asse di simmetria verticale del problema, è stato applicato seguendo una rampa di carico e scarico (Fig. 5): ogni step di carico aveva una durata temporale di 15 secondi, ottenendo, così, la durata totale di una prova Vickers standard.

Infine, si può concludere il presente paragrafo osservando che il comportamento elasto-plastico del materiale su cui veniva eseguita l'indentazione è stato sempre descritto in ambiente ANSYS $^{\circledR}$ assumendo come criterio di incrudimento un incrudimento di tipo isotropico.

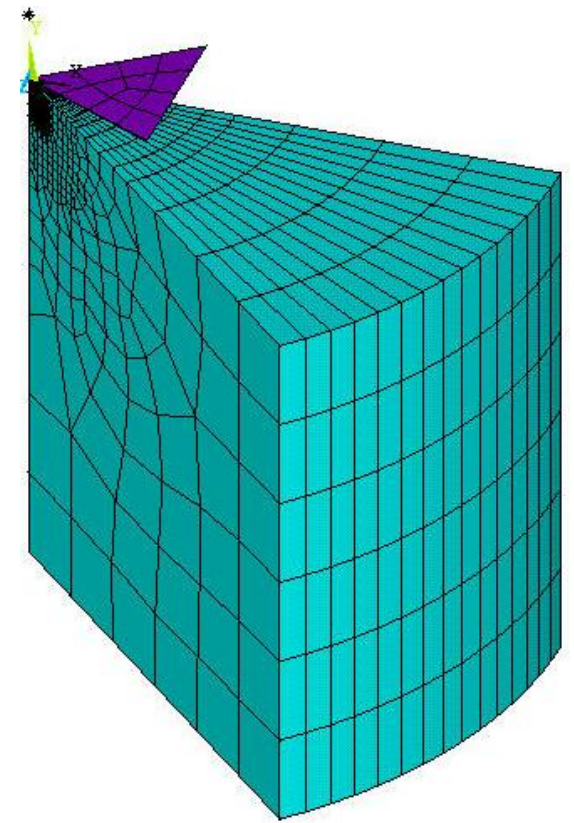

(a)

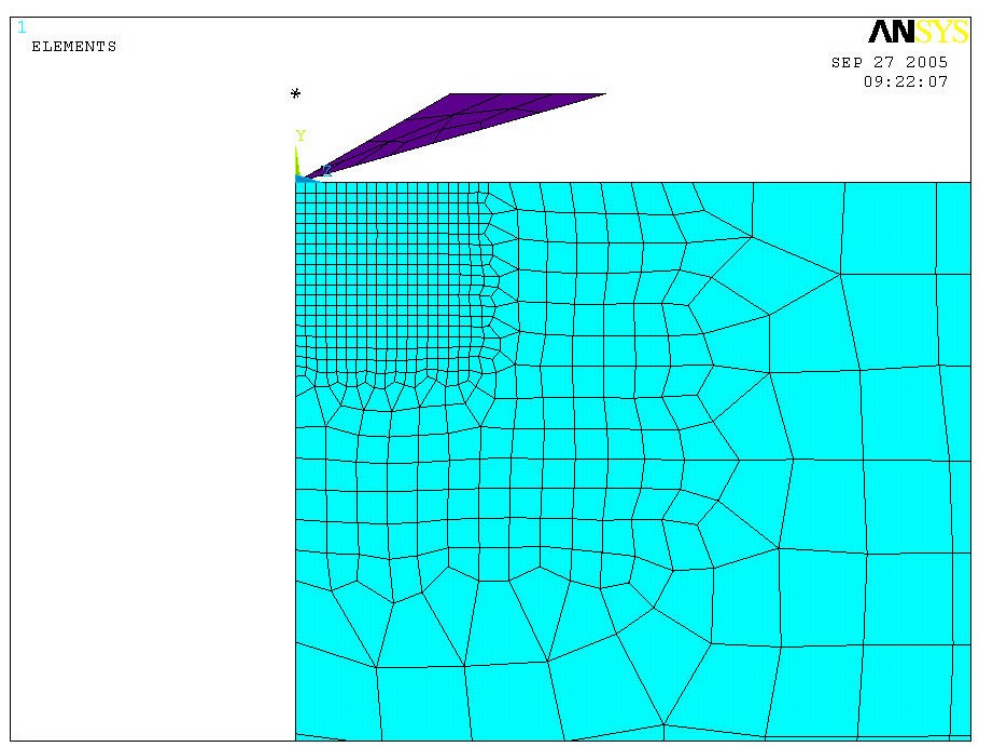

(b)

Figura 3: Distribuzione della mesh nella simulazione agli elementi finiti del processo di indentazione eseguita mediante il software ANSYS ${ }^{\circ}$.

Figure 3: Mesh distribution in the FE models used to simulate the Vickers test.
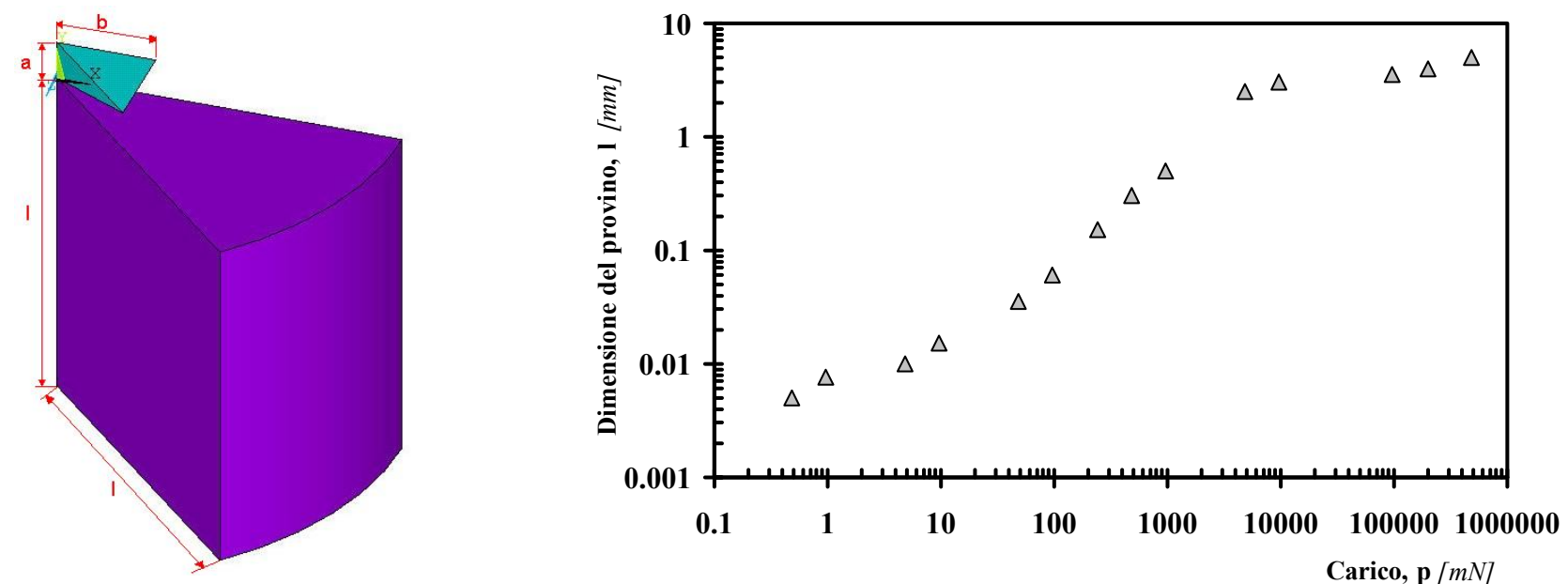

Figura 4: Criterio utilizzato per determinare la dimensione, l, del provino da modellare in ambiente ANSYS® al variare del carico applicato all'indentatore.

Figure 4: Definition of dimension 1 used to do the FE models of the Vickers indenters. 


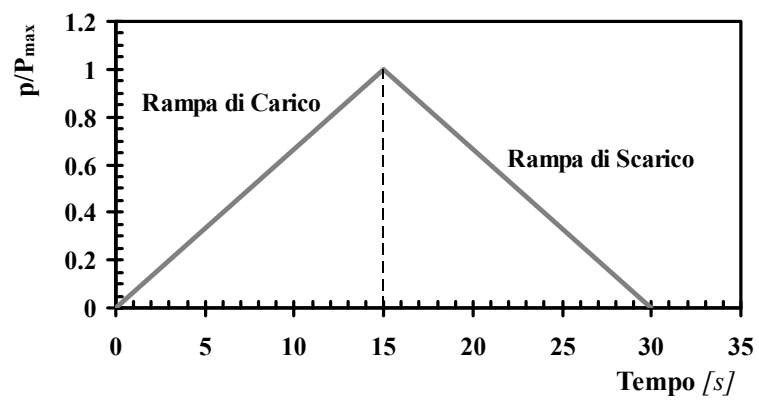

Figura 5: Rampa di carico e scarico utilizzata per simulare una prova Vickers standard.

Figure 5: Loading and unloading curve used to simulate the Vickers test.

\section{RISULTATI SPERIMENTALI DELLE MISURE DI MACRO-, MICRO- E NANO-DUREZZA}

$\mathrm{L}$ e misure di macro-, micro- e nano-durezza sono state eseguite su tre materiali metallici con caratteristiche metallurgiche alquanto diverse: un acciaio a basso tenore di carbonio (BS970-En3B), un acciaio inossidabile (AISI 316L) e, infine, una lega d'alluminio (7075-T6). Le proprietà meccaniche di questi metalli, e in particolare le curve sforzo-deformazione in campo elasto-plastico, sono state ricavate con prove eseguite in accordo alle vigenti normative. Come verrà ampiamente descritto nei paragrafi successivi, per investigare in modo più accurato l'influenza della dimensione della superficie indentata sul valore della durezza, le analisi FEM relative alle prove condotte sull'acciaio inossidabile AISI 316L sono state calibrate non solo considerando la curva elasto-plastica ottenuta mediante provini di dimensione convenzionale, ma anche mediante quella ottenuta con provini aventi larghezza della zona calibra dell'ordine dei $100 \mu \mathrm{m}$, ovvero dell'ordine dei 10 grani [14].

Le principali proprietà meccaniche dei materiali investigati sono riassunte nella Tab. 1, mentre nel grafico di Fig. 6 sono comparate le curve elasto-plastiche ottenute dalle prove di trazione, curve che sono state tracciate in tale diagramma a mezzo delle corrispondenti relazioni di Ramberg-Osgood [11]:

$$
\varepsilon_{\mathrm{tot}}=\varepsilon_{\mathrm{el}}+\varepsilon_{\mathrm{pl}}=\frac{\sigma_{\mathrm{t}}}{\mathrm{E}}+\left(\frac{\sigma_{\mathrm{t}}}{\mathrm{K}}\right)^{1 / \mathrm{n}}
$$

\begin{tabular}{ccccccccc}
\hline Materiale & $\mathbf{E}$ & $\boldsymbol{\sigma}_{\mathbf{Y}}$ & $\boldsymbol{\sigma}_{\mathbf{U T S}}$ & $\boldsymbol{\sigma}_{\mathbf{f}}$ & $\boldsymbol{\varepsilon}_{\mathbf{f}}$ & $\mathbf{K}$ & $\mathbf{n}$ & $\begin{array}{c}\text { Grain Size } \\
{[\mu \mathrm{m}]}\end{array}$ \\
& {$[\mathrm{MPa}]$} & {$[\mathrm{MPa}]$} & {$[\mathrm{MPa}]$} & {$[\mathrm{MPa}]$} & & {$[\mathrm{MPa}]$} & & 10 (transv.) \\
7075-T6 & 65000 & 500.5 & 577 & 624.3 & 0.08 & 709.1 & 0.048 & 13 \\
BS970 En3B & 197400 & 606.2 & 638 & 851.8 & 0.56 & 882.7 & 0.061 & 12 \\
AISI 316L & 177000 & 298.8 & 754 & 986.5 & 0.39 & 1238.5 & 0.251 & 12 \\
AISI 316L (wire) & 150000 & 315.1 & 580 & - & - & 1252.3 & 0.346 & \\
\hline
\end{tabular}

Tabella 1: Proprietà meccaniche dei materiali metallici considerati nella presente investigazione.

Table 1: Mechanical properties of the investigated metallic materials.

I valori delle costanti della precedente relazione sono riportati, per ogni materiale considerato, in Tab. 1.

Le misure di durezza superficiale sono state eseguite utilizzando tre diversi strumenti di misura: un macro-durometro convenzionale, un micro-durometro Mitutoyo MVK-H1 ed un Nanoindenter XP-MTS, tutti equipaggiati con punte Vickers standard. Le rilevazioni sperimentali sono state eseguite a temperatura ambiente controllata, e pari a $20^{\circ} \mathrm{C}$, su provini preparati in accordo con le normative vigenti e lucidati a specchio con pasta diamantata da $1 \mu \mathrm{m}$.

I grafici di Fig. 7 sintetizzano i risultati delle misure di durezza eseguite sui tre materiali metallici considerati nella presente investigazione: ognuno dei punti sperimentali riportato in tali diagrammi rappresenta il valore medio ricavato da sei misurazioni eseguite per ciascuna condizione di carico considerata. 


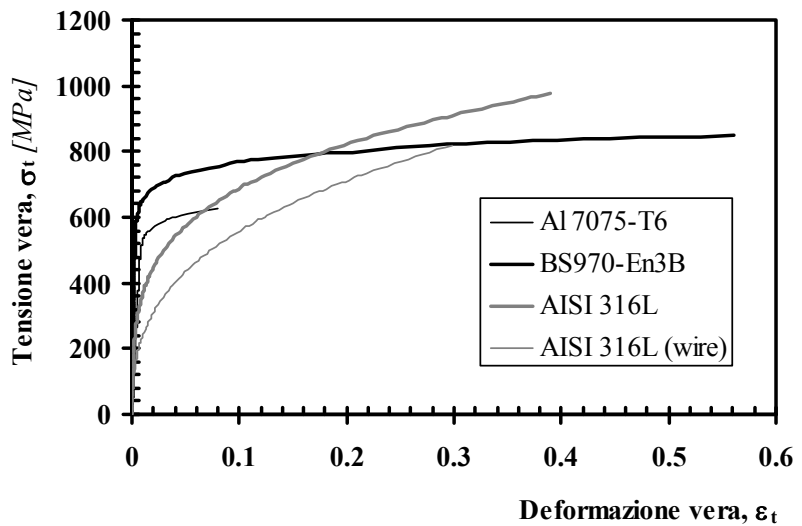

Figura 6: Leggi costitutive dei materiali investigati espresse in termini di tensioni e deformazioni vere e tracciate in accordo alla relazione di Ramberg-Osgood.

Figure 6: True stress vs. true strain curves of the investigated materials.
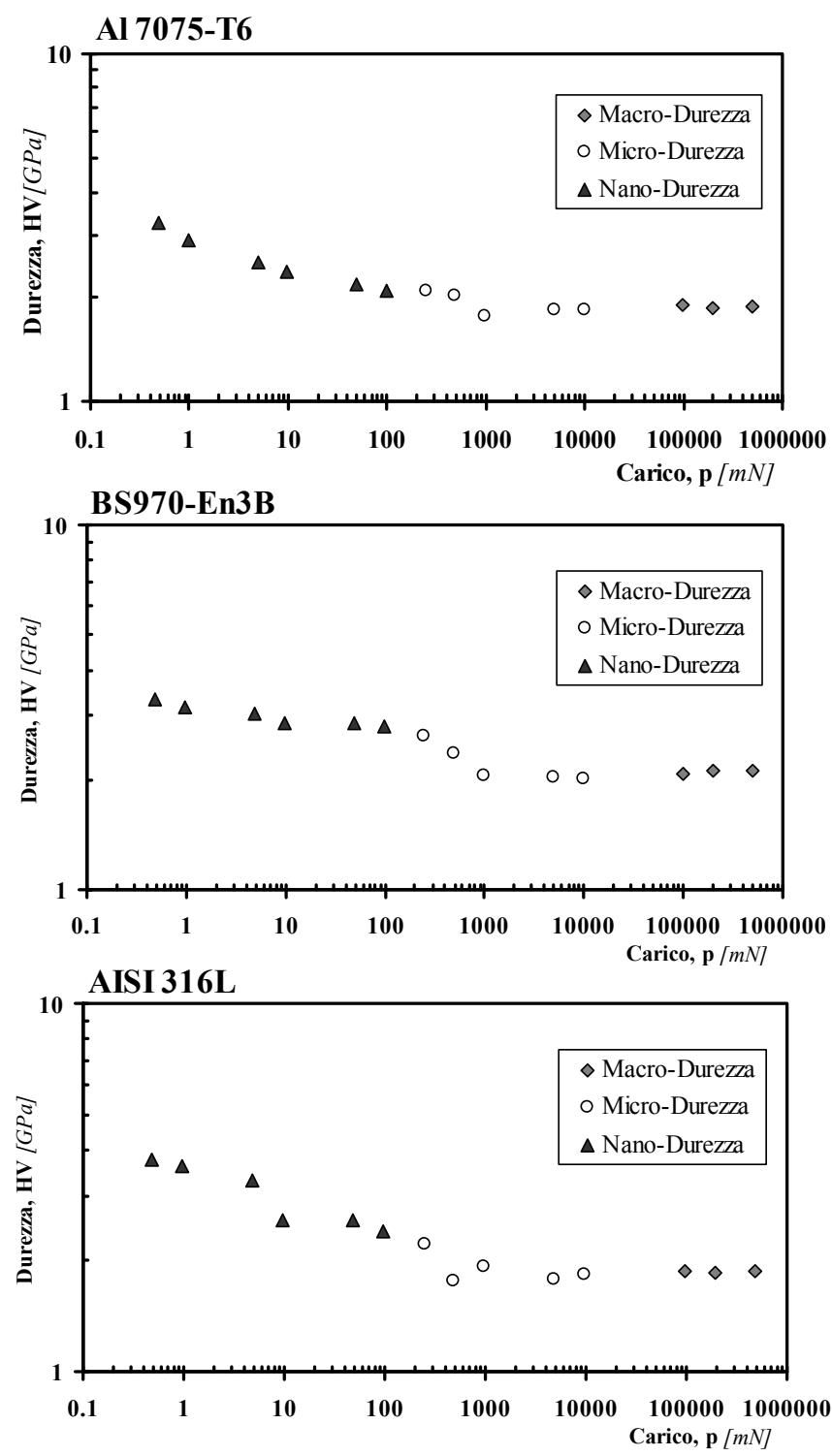

Figura 7: Risultati delle prove di durezza Vickers eseguite sui diversi materiali investigati.

Figure 7: Results of the Vickers tests. 
Si può subito osservare come le macro-prove Vickers mostrino, per tutti e tre i materiali, un valore di durezza sostanzialmente indipendente dal carico di prova, e questo ad ulteriore conferma di quanto già da tempo noto [5].

Nel zona della microdurezza invece, i valori misurati tendono, per tutti materiali considerati, a crescere al decrescere del carico di prova applicato, e questo incremento diventa chiaramente evidente in corrispondenza dei limiti inferiori di carico delimitanti la zona di utilizzo del micro-durometro. Importante è anche osservare che in questa zona dei diagrammi durezza-carico si è sempre osservato un anomalo comportamento dei risultati sperimentali. Analisi comparative hanno chiaramente evidenziato che questa anomalia poteva essere imputata ad un problema di cambio di scala nella catena di misura del micro-durometro, cambio di scala che avveniva, per carichi inferiori a $1 \mathrm{~N}$, quando la forza al penetratore passava dall'essere applicata mediante contrappesi all'essere applicata per effetto elettromagnetico. Fortunatamente la sovrapposizione del campo superiore di utilizzo del nano-durometro con quella inferiore del micro-durometro ha permesso di limitare questo problema, ottenendo così risultati più coerenti.

Infine, i diagrammi di Fig. 7 mostrano che il raggiungimento dei limiti inferiori di carico nel campo delle misure di nanodurezza corrispondeva ad un marcato incremento del valore della durezza rispetto a quella misurata in campo macroscopico (questo trend può essere apprezzato con maggior dettaglio osservando la Tab. 2).

A conclusione di questo paragrafo, si può da ultimo evidenziare che nel campo delle nano-indentazioni i valori di durezza misurati risultavano sempre caratterizzati da una dispersione statistica, in termini di deviazione standard, più elevata rispetto ai valori di micro- e macro-durezza, e questo in conseguenza al ruolo fondamentale giocato dalla reale morfologia del materiale in presenza di superfici indentate di estensione inferiore alla dimensione del grano.

\begin{tabular}{cccc}
\hline & \multicolumn{2}{c}{ Durezza HV } & Incremento \\
Materiale & $\mathbf{p}=\mathbf{4 9 0} \mathbf{N}$ & $\mathbf{p}=\mathbf{0 . 4 9} \mathbf{~ m N}$ & \\
{$[G P a]$} & {$[G P a]$} & {$[\%]$} \\
Al 7075T6 & 1.88 & 3.25 & 72.8 \\
BS970 En3B & 2.11 & 3.34 & 58.3 \\
AISI 316L & 1.87 & 3.75 & 100.5 \\
\hline
\end{tabular}

Tabella 2: Incremento delle durezze misurate in campo microscopico rispetto a quelle determinate in campo macroscopico.

Table 2: Comparison between measured micro- and macro- harness.

\section{COMPARAZIONE TRA MISURE SPERIMENTALI E SIMULAZIONI NUMERICHE}

T a Tab. 3 sintetizza i valori dell'errore commesso nello stimare, mediante l'approccio agli elementi finiti discusso in precedenza, la durezza dei tre materiali considerati nella presente investigazione. Importante è subito sottolineare come tutte le stime riportate nella sopramenzionata Tabella siano state ottenute assumendo che i materiali metallici considerati obbedissero alle leggi della meccanica del continuo e che il loro comportamento elasto-plastico fosse descritto da curve monotone ottenute con provini convenzionali (ovvero, macroscopici).

La Tab. 3 pone, prima di tutto, in evidenza come i valori ricavati dalle simulazioni di macro-durezza siano tutti in perfetto accordo con le rilevazioni sperimentali. In particolare, il fatto che l'errore massimo sia sempre inferiore al 3\%, sembra fortemente supportare l'idea che la durezza in campo macroscopico possa essere efficacemente stimata facendo semplicemente uso della classica meccanica del continuo.

I risultati di Tab. 3 mostrano poi come al diminuire del carico di prova, ovvero spostandosi dal campo della macrodurezza al campo della micro-durezza, l'errore nella stima rimanga contenuto, ma in evidente tendenza alla crescita. Quando, infine, si entra decisamente nel campo della nano-durezza, l'errore nella stima cresce notevolmente, arrivando anche a valori superiori al $50 \%$.

Interessante è, prima di tutto, osservare come però l'errore commesso nella stima della nano-durezza dell'acciaio da costruzione BS970-En3B sia risultato essere molto basso, ovvero con errori, indipendentemente dall'entità del carico applicato, sempre inferiori all'10\%. Una tale situazione può essere spiegata con semplicità, osservando che le barre di BS970-En3B da cui erano stati estratti sia i provini per le misure di durezza che i provini per le prove statiche sono state ottenute per rollatura a freddo: il materiale si presentava, pertanto, già fortemente incrudito e questo limitava notevolmente l'effetto della strain gradient plasticity [10] sul valore della durezza, visto che un ulteriore moto delle dislocazioni risultava altamente inibito a causa della precedente lavorazione a freddo. 


\begin{tabular}{|c|c|c|c|c|c|}
\hline \multirow{3}{*}{ Materiale } & \multirow{3}{*}{ Scala } & \multirow{3}{*}{$\begin{array}{l}\text { Carico } \\
{[\mathrm{mN}]}\end{array}$} & \multicolumn{2}{|c|}{ HV } & \multirow{3}{*}{$\begin{array}{c}\text { Errore } \\
{[\%]}\end{array}$} \\
\hline & & & Calcolata & Misurata & \\
\hline & & & [GPa] & [GPa] & \\
\hline \multirow{14}{*}{ 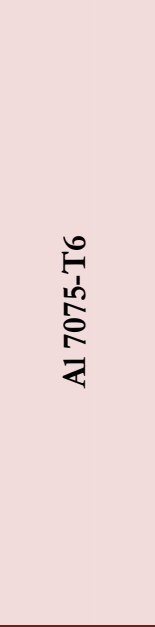 } & \multirow{3}{*}{$\begin{array}{l}0 \\
\stackrel{0}{J} \\
\sum\end{array}$} & 490000 & 1.84 & 1.88 & 2.2 \\
\hline & & 196000 & 1.83 & 1.86 & 1.6 \\
\hline & & 98000 & 1.87 & 1.89 & 1.1 \\
\hline & \multirow{5}{*}{ 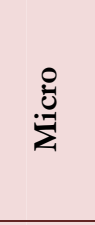 } & 9800 & 1.75 & 1.83 & 4.6 \\
\hline & & 4900 & 1.75 & 1.83 & 4.6 \\
\hline & & 980 & 1.59 & 1.76 & 10.7 \\
\hline & & 490 & 1.75 & 2.01 & 14.9 \\
\hline & & 245 & 1.77 & 2.08 & 17.5 \\
\hline & \multirow{6}{*}{$\begin{array}{l}\stackrel{8}{\pi} \\
\text { Z }\end{array}$} & 98 & 1.89 & 2.07 & 9.5 \\
\hline & & 49 & 2.07 & 2.16 & 4.3 \\
\hline & & 9.8 & 2.14 & 2.37 & 10.7 \\
\hline & & 4.9 & 2.09 & 2.5 & 19.6 \\
\hline & & 0.98 & 2.13 & 2.92 & 37.1 \\
\hline & & 0.49 & 2.14 & 3.25 & 51.9 \\
\hline \multirow{14}{*}{ 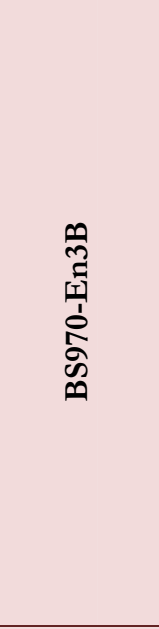 } & \multirow{3}{*}{ 冚 } & 490000 & 2.07 & 2.11 & 1.9 \\
\hline & & 196000 & 2.08 & 2.12 & 1.9 \\
\hline & & 98000 & 2.08 & 2.07 & -0.5 \\
\hline & \multirow{5}{*}{ : } & 9800 & 1.97 & 2.01 & 2.0 \\
\hline & & 4900 & 2.09 & 2.04 & -2.4 \\
\hline & & 980 & 1.99 & 2.05 & 3.0 \\
\hline & & 490 & 2.25 & 2.36 & 4.9 \\
\hline & & 245 & 2.5 & 2.63 & 5.2 \\
\hline & \multirow{6}{*}{$\begin{array}{l}\stackrel{8}{\Xi} \\
\text { Z }\end{array}$} & 98 & 2.65 & 2.8 & 5.7 \\
\hline & & 49 & 2.7 & 2.85 & 5.6 \\
\hline & & 9.8 & 2.75 & 2.87 & 4.4 \\
\hline & & 4.9 & 2.94 & 3.04 & 3.4 \\
\hline & & 0.98 & 3.04 & 3.15 & 3.6 \\
\hline & & 0.49 & 3.08 & 3.34 & 8.4 \\
\hline \multirow{14}{*}{ 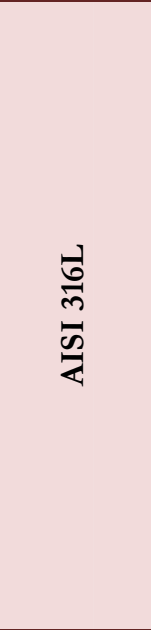 } & \multirow{3}{*}{ 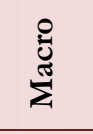 } & 490000 & 1.83 & 1.87 & 2.2 \\
\hline & & 196000 & 1.83 & 1.86 & 1.6 \\
\hline & & 98000 & 1.84 & 1.87 & 1.6 \\
\hline & \multirow{5}{*}{ : } & 9800 & 1.75 & 1.84 & 5.1 \\
\hline & & 4900 & 1.75 & 1.79 & 2.3 \\
\hline & & 980 & 1.75 & 1.93 & 10.3 \\
\hline & & 490 & 1.84 & 1.77 & -3.8 \\
\hline & & 245 & 1.88 & 2.22 & 18.1 \\
\hline & \multirow{6}{*}{ 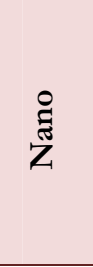 } & 98 & 2.18 & 2.41 & 10.6 \\
\hline & & 49 & 2.18 & 2.58 & 18.3 \\
\hline & & 9.8 & 2.18 & 2.59 & 18.8 \\
\hline & & 4.9 & 2.19 & 3.32 & 51.6 \\
\hline & & 0.98 & 2.22 & 3.63 & 63.5 \\
\hline & & 0.49 & 2.2 & 3.75 & 70.5 \\
\hline
\end{tabular}

Tabella 3: Comparazione tra i valori di durezza HV ottenuti sperimentalmente e quelli ottenuti mediante analisi numeriche agli elementi finiti.

Table 3: Comparison between the experimental values of the Vickers hardness and the corresponding values determined by solving elasto-plastic FE models. 
In accordo con quanto riportato in Tab. 3, si può, pertanto, affermare che la tendenza evidenziata dall'errore nella stima della durezza (passando dal macro- al nano-) sembra fortemente supportare l'idea che la meccanica del continuo possa fornire risultati soddisfacenti fintanto che la dimensione dell'impronta resta maggiore della dimensione media della grana cristallina. Al contrario, quando l'impronta presenta una estensione confrontabile, o inferiore, alla dimensione media del grano, è evidente che l'ipotesi di perfetta omogeneità ed isotropia del materiale, ipotesi su cui è basata la meccanica del continuo, risulta essere troppo semplicistica. In altre parole, quando l'area della superficie indentata diventa confrontabile con la dimensione del grano, la reale morfologia del materiale ne influenza troppo il comportamento meccanico per essere completamente trascurata nella modellazione del fenomeno.

Alla luce delle considerazioni riportate sopra, per verificare se si poteva comunque tenere conto correttamente della influenza della struttura cristallina del materiale restando, però, ancora nell'ambito della meccanica del continuo, la durezza dell'acciaio inossidabile AISI 316L è stata stimata anche a mezzo di analisi FEM calibrate mediante la curva monotona del materiale ottenuta a mezzo di provini microscopici, ovvero di provini aventi larghezza della zona calibra pari a circa 100 $\mu \mathrm{m}$ [14]. Il diagramma di Fig. 6 mostra chiaramente che la curva ottenuta con provini microscopici (wire) differiva dalla corrispondente curva monotona generata con provini di dimensione convenzionale. Questa diversità deve essere imputata al fatto che nel caso dei provini microscopici la reale morfologia del materiale nella zona di processo ricopriva un ruolo fondamentale nella definizione del profilo della curva monotona stessa. Al contrario, nei macro-provini la reale distribuzione dei grani giocava, invece, un ruolo del tutto marginale a causa dei noti fenomeni di omogeneità ed isotropia di compenso tipica dei materiali metallici.

In Tab. 4 sono stati sintetizzati gli errori ottenuti nello stimare la durezza dell'acciaio inossidabile AISI 316L, quando i modelli FEM venivano calibrati con la curva monotona ottenuta con micro-provini. Sfortunatamente, questo tentativo, se pure estremamente attraente da un punto di vista filosofico, non ha dato i risultati sperati, portando ad errori nella stima comunque superiori a quelli che si erano ottenuti calibrando i modelli FEM con la curva tensione-deformazione generata con provini macroscopici.

\begin{tabular}{|c|c|c|c|c|c|}
\hline \multirow[b]{2}{*}{ Materiale } & \multirow[b]{2}{*}{ Scala } & \multirow[b]{2}{*}{$\begin{array}{c}\text { Carico } \\
{[\mathrm{mN}]}\end{array}$} & \multicolumn{2}{|c|}{ HV } & \multirow{2}{*}{$\begin{array}{c}\text { Errore } \\
{[\%]}\end{array}$} \\
\hline & & & $\begin{array}{c}\text { Calcolata } \\
{[G P a]}\end{array}$ & $\begin{array}{c}\text { Misurata } \\
{[G P a]}\end{array}$ & \\
\hline \multirow{11}{*}{ 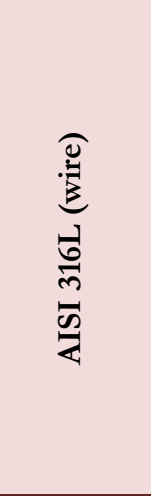 } & \multirow{5}{*}{ 일 } & 9800 & 1.50 & 1.84 & 22.7 \\
\hline & & 4900 & 1.49 & 1.79 & 20.1 \\
\hline & & 980 & 1.32 & 1.93 & 46.2 \\
\hline & & 490 & 1.35 & 1.77 & 31.1 \\
\hline & & 245 & 1.36 & 2.22 & 63.2 \\
\hline & \multirow{6}{*}{ Z } & 98 & 1.71 & 2.41 & 40.9 \\
\hline & & 49 & 1.67 & 2.58 & 54.5 \\
\hline & & 9.8 & 1.69 & 2.59 & 53.3 \\
\hline & & 4.9 & 1.74 & 3.32 & 90.8 \\
\hline & & 0.98 & 1.76 & 3.63 & 106.3 \\
\hline & & 0.49 & 1.75 & 3.75 & 114.3 \\
\hline
\end{tabular}

Tabella 4: Comparazione tra i valori di durezza HV ottenuti sperimentalmente e quelli ottenuti mediante analisi numeriche agli elementi finiti, calibrando queste ultime mediante la curva monotona ottenuta testando provini di dimensione microscopica.

Table 4: Comparison between the experimental values of the Vickers hardness and the corresponding values determined by solving elasto-plastic FE models calibrated through the Ramberg-Osgood curve generated by testing microscopic samples of AISI 316L.

A conclusione di quanto riportato nel presente paragrafo, si può rimarcare come la durezza superficiale dei materiali metallici possa essere stimata correttamente ricorrendo alla sola meccanica del continuo fintanto che la dimensione della superficie indentata risulta essere notevolmente maggiore della dimensione media della grana cristallina. Al contrario, per dimensioni dell'impronta comparabili, o inferiori, alla dimensione della grana cristallina, la plasticità classica risulta in una sottostima sia della micro- che della nano-durezza. Da ultimo si può evidenziare che, in accordo alle analisi numeriche e sperimentali condotte sull'AISI 316L, neanche l'impiego di una curva monotona generata con provini di larghezza dell'ordine di pochi grani consente di estendere con successo l'utilizzo della meccanica del continuo fino ad un livello microscopico: in questo scenario così complesso e articolato si comprende, pertanto, il motivo per cui nell'ultimo 
decennio gli specialisti della cosiddetta "surface science" abbiano investito così tante risorse nello sviluppare, e validare sperimentalmente, la teoria dello strain gradient plasticity [8-10].

\section{UNA PROCEDURA SEMPLIFICATA PER LA CORREZIONE DEI RISULTATI NUMERICI ONDE INCREMENTARE LA PRECISIONE NELLA STIMA DI MICRO- E NANO-DUREZZE}

$\mathrm{N}$

el paragrafo precedente, confrontando i risultati sperimentali con i valori ottenuti dalle simulazioni numeriche, si è potuto mettere in luce che l'errore nella previsione aumentava al diminuire della dimensione dell'impronta. In particolare, si è potuto osservare come un approccio agli elementi finiti, e basato sulla teoria della meccanica del continuo, consentisse di prevedere con elevata accuratezza solo il valore della durezza misurata in campo macroscopico, tendendo, sfortunatamente, a diventare sempre meno accurato mano a mano che la dimensione della superficie indentata tendeva a diventare confrontabile con la dimensione media della grana cristallina.

In accordo con le più recenti teorie sviluppate nel campo della "surface science", anche gli autori del presente lavoro ritengono che la via più promettente per tentare di incrementare la precisione nella stima della durezza sia quella di sviluppare algoritmi numerici che siano in grado ti tenere conto in modo efficace dell'effetto della strain gradient plasticity [810] sulla generazione dell'impronta indentata. Tuttavia, nonostante questa convinzione, in questo paragrafo si cerca di propone una semplice metodologia ingegneristica adatta a correggere i valori della durezza superficiale quando questi sono stimati con un approccio agli elementi finiti.

Inizialmente, osservando la Tab. 3, si può constatare come l'accuratezza più elevata nella stima della durezza in campo nano-scopico si sia ottenuta considerando i risultati generati dalle prove sull'acciaio al carbonio BS970-En3B. Si può poi osservare che la curva monotona di questo materiale (Fig. 6) presentava, a causa della lavorazione a freddo con cui erano state ottenute le barre del materiale base, un tratto plastico praticamente orizzontale, ovvero il comportamento di tale materiale poteva, di fatto, essere assimilato ad un comportamento di tipo "perfettamente elastico-perfettamente plastico". Da un punto di vista della fisica del fenomeno, una tale curva denotava, invece, il fatto che il materiale, a causa della lavorazione a freddo, aveva già subito un forte incrudimento: il moto residuo delle dislocazioni risultava alquanto limitato, riducendo gli effetti della strain gradient plasticity sul valore misurato della durezza.

Alla luce delle osservazioni riportate appena sopra si è formulata allora l'ipotesi che l'effetto della strain gradient plasticity sul valore della durezza rilevata potesse essere assunto come proporzionale ad una sorta di "gradiente equivalente del comportamento plastico macroscopico" definito come:

$$
\frac{\Delta \sigma}{\Delta \varepsilon}=\frac{\sigma_{\mathrm{f}}-\sigma_{\mathrm{Y}}}{\varepsilon_{\mathrm{f}}-\varepsilon_{\mathrm{Y}}}=\frac{\sigma_{\mathrm{f}}-\sigma_{\mathrm{Y}}}{\varepsilon_{\mathrm{f}}-\frac{\sigma_{\mathrm{Y}}}{\mathrm{E}}}
$$

In base alla definizione (3) un materiale ideale avente un comportamento perfettamente plastico sarebbe caratterizzato, allora, da un rapporto $\Delta \sigma / \Delta \varepsilon$ pari a zero, ovvero, in accordo con le considerazioni esposte sopra, il contributo della strain gradient plasticity sul valore della durezza dovrebbe essere di fatto trascurabile. Al contrario, se fosse vero quanto ipotizzato poco sopra, al crescere del valore del rapporto espresso dalla relazione (3), dovrebbe crescere il contributo del gradiente della deformazione sul valore finale della durezza.

Importante è poi osservare che le costanti del materiale necessarie per la determinazione del rapporto $\Delta \sigma / \Delta \varepsilon$ sono, per come è stato posto il problema, sempre note: tali costanti, infatti, vengono dalla conoscenza della curva monotona del materiale, curva che deve essere nota perché necessaria per eseguire le analisi FEM in campo elasto-plastico.

In base a quanto ipotizzato, diventa, a questo punto, lecito assumere che il valore misurato sperimentalmente della durezza, $\mathrm{HV}_{\mathrm{s}}$, possa essere previsto, noto il corrispondente valore ottenuto dalle simulazione agli elementi finiti, $\mathrm{HV}_{\mathrm{FEM}}$, e il valore p del carico applicato all'indentatore, mediante la seguente relazione:

$$
\frac{\mathrm{HV}}{\mathrm{HV}} \mathrm{FEM}_{\mathrm{FE}} \mathrm{p}^{\alpha}=\mathrm{Q}=\cos \mathrm{t}
$$

Una conveniente espressione per l'esponente $\alpha$ è risultata essere la seguente:

$$
\alpha=10^{-5} \frac{\Delta \sigma}{\Delta \varepsilon}
$$

dove, chiaramente, $\alpha$ è una quantità resa adimensionale dalla costante $10^{-5}$ misurata in $\mathrm{MPa}^{-1}$. 

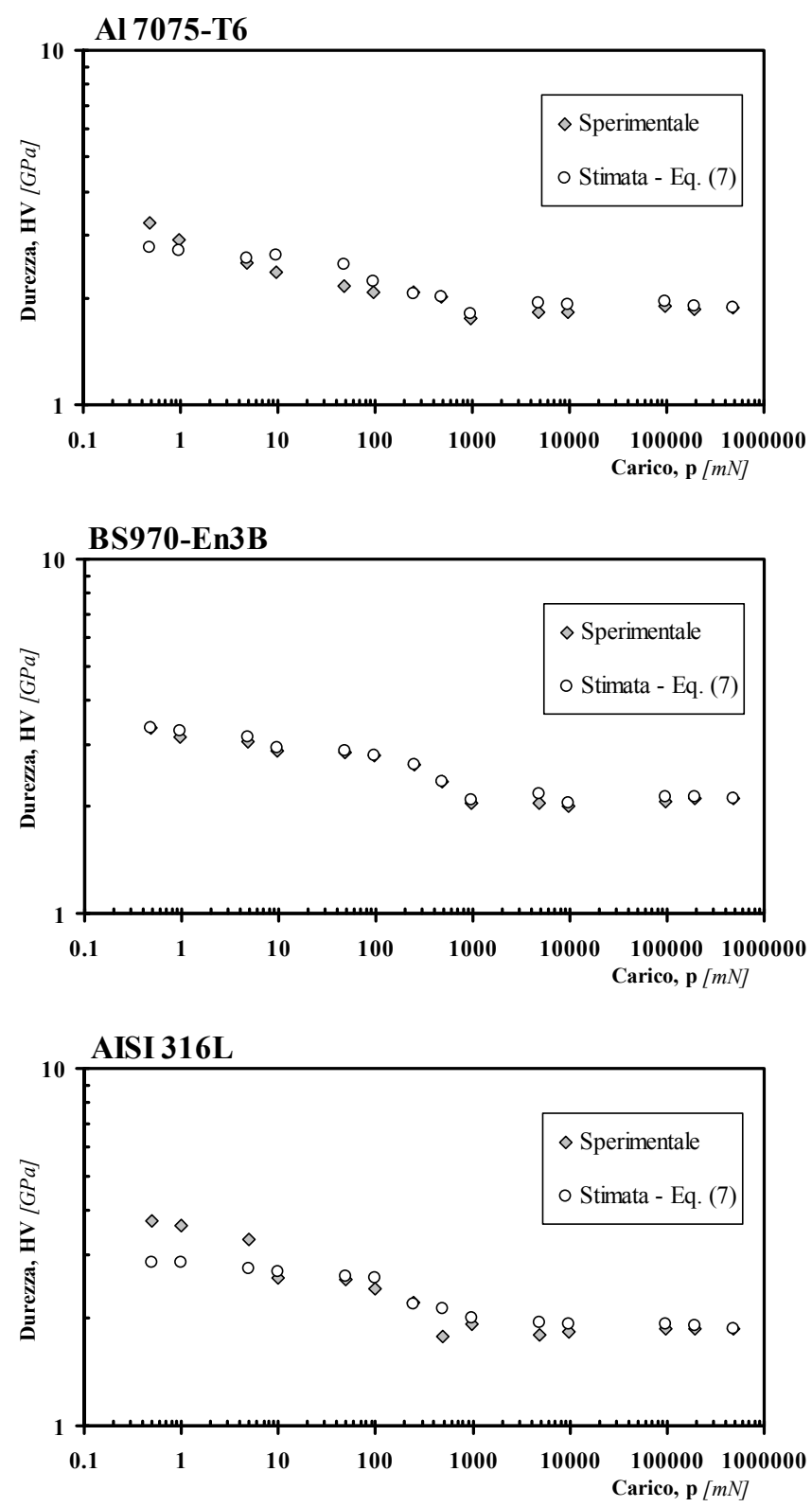

Figura 8: Comparazione tra risultati sperimentali e stime FEM corrette a mezzo della metodologia proposta.

Figure 8: Comparison between experimental results and predictions made by correcting the calculated FE values according to the proposed engineering method.

Il valore della costante Q nella relazione (4) può essere determinato considerando una configurazione di riferimento per cui siano noti i valori dei tre parametri $\mathrm{HV}_{\mathrm{S}}, \mathrm{HV}_{\mathrm{FEM}} \mathrm{e} \mathrm{p}$ :

$$
\mathrm{Q}=\left.\frac{\mathrm{HV}_{\mathrm{S}}}{\mathrm{HV} \mathrm{V}_{\mathrm{FEM}}} \cdot \mathrm{p}^{\alpha}\right|_{\text {Rif }}=\cos \mathrm{t}
$$

Pertanto per un qualsiasi valore del carico applicato all'indentatore, e una volta calcolato il valore di HVFEM mediante un modello agli elementi finiti, la durezza del materiale può essere stimata come:

$$
\mathrm{HV}_{\mathrm{S}}=\mathrm{Q} \cdot \frac{\mathrm{HV}_{\mathrm{FEM}}}{\mathrm{p}^{\alpha}}
$$


I risultati ottenuti applicando la metodologia ingegneristica proposta in questo paragrafo sono riassunti nei diagrammi di Fig. 8, dove tali diagrammi sono stati ottenuti assumendo, per tutti i materiali, come configurazione di riferimento per il calcolo della costante Q, Eq. (6), quella relativa ad una prova di macro-durezza dove il carico applicato all'indentatore era pari a $490 \mathrm{~N}$.

Si può allora concludere questo paragrafo evidenziando come, in base a quanto mostrato dalla Fig. 8, l'approccio proposto, seppure estremamente semplificato, consente di ottenere delle stime della durezza superficiale che sono comunque sufficientemente accurate da un punto di vista ingegneristico e quindi la metodologia di stima sviluppata presenta una indubbia utilità pratica.

\section{Discussione}

I 1 lavoro sintetizzato nel presente articolo si poneva come obbiettivo primario quello di verificare se un approccio elasto-plastico agli elementi finiti fosse in grado di stimare in modo ingegneristicamente accurato la durezza dei materiali metallici convenzionali, e questo sia a livello macroscopico, che a livello microscopico, che, infine, a livello nanoscopico.

Per verificare validità e accuratezza della metodologia FEM messa a punto, sono state condotte una serie di analisi sperimentali su tre materiali metallici (Al 7075-T6, BS970-En3B e AISI 316L) aventi caratteristiche metallurgiche alquanto diverse. Una tale investigazione sperimentale ha inizialmente evidenziato, confermando, d'altra parte, quanto già noto in letteratura, come il valore della durezza Vickers (o più ingenerale della durezza misurata a mezzo di un indentatore) tenda a crescere al diminuire della dimensione della impronta: per uno stesso materiale, $i$ valori di durezza misurati in campo nanoscopico sono risultati anche superiori del 100\% ai valori determinati in campo macroscopico.

Una tale diversità può essere imputata al diverso ruolo giocato dalla dimensione del grano sul comportamento meccanico globale del materiale, ovvero al diverso ruolo giocato dalla reale morfologia del materiale sul valore della durezza misurata al diminuire delle dimensioni della superficie indentata. In particolare, e come noto, per i materiali metallici valgono, in campo macroscopico, le ipotesi di omogeneità e di isotropia di compenso grazie alle quali l'influenza della grana cristallina può essere totalmente trascurata nel modellare i fenomeni in presenza di grandi deformazioni plastiche: in questo ambito la meccanica del continuo risulta idonea a descrivere il fenomeno investigato, ovvero analisi FEM condotte in campo elasto-plastico, ipotizzando un incrudimento di tipo isotropico, consentono di stimare con estrema accuratezza il valore dell'area della superficie indentata.

Al contrario, al diminuire della dimensione della superficie indentata, il ruolo della struttura cristallina diventa sempre più importante, tanto che quando l'area dell'impronta diventa confrontabile con la dimensione media del grano, la meccanica del continuo, applicata con metodologie FEM, non sembra essere più in grado di modellare correttamente il fenomeno investigato.

Nel tentativo di verificare se esisteva comunque la possibilità di estendere l'uso della meccanica del continuo anche al campo nanoscopico, si è provato a stimare la durezza superficiale dell'AISI 316L anche utilizzando per la calibrazione dei modelli FEM la curva monotona ottenuta testando provini aventi larghezza della zona calibra dell'ordine dei $100 \mu \mathrm{m}$. Questo tentativo prendeva come spunto di partenza l'idea che un approccio agli elementi finiti potesse essere esteso con successo anche a livello nanoscopico, ammesso che per la sua calibrazione si potessero utilizzare proprietà del materiale il più possibile vicine a quelle relative al singolo grano. Purtroppo, però, tale tentativo, anche se molto interessante dal punto di vista filosofico/teorico, non ha dato i risultati sperati. A parere degli scriventi, il motivo di un tale insuccesso può essere ricondotto principalmente al fatto che il comportamento meccanico di un agglomerato cristallino, anche se formato da pochi grani, differisce comunque dal comportamento meccanico di un singolo grano. In più, è evidente che quando l'area della superficie indentata risulta essere inferiore alla dimensione del grano le reali proprietà meccaniche/metallurgiche del grano stesso influenzano necessariamente in modo determinante il valore misurato della durezza. A questo livello, l'orientazione delle direzioni preferenziali di scorrimento delle dislocazioni, nonché la possibilità che hanno tali dislocazioni di muoversi in presenza di fenomeni di deformazione così spinti e localizzati, influenza in modo decisivo le caratteristiche dell'impronta generata dall'indentatore.

Alla luce di queste considerazioni si comprende il motivo per cui la teoria dello strain gradient plasticity [10] sia oggi quella maggiormente utilizzata per spiegare in modo rigoroso e scientifico i fenomeni investigati nel presente lavoro: a livello nanoscopico, il moto delle dislocazioni in presenza di forti gradienti di deformazione gioca il ruolo più importante nella definizione della durezza, quando questa è misurata a mezzo di un indentatore. Nonostante questa convinzione però, nel presente lavoro si è comunque tentato di formalizzare una regola pratica, basata sul comportamento plastico del materiale in campo macroscopico, nel tentativo di proporre uno strumento ingegneristico che consentisse di stimare la durezza, 
partendo da un valore stimato mediante gli elementi finiti, indipendentemente dalla dimensione dell'impronta. La comparazione diretta tra stime e risultati sperimentali ha consentito di evidenziare come la metodologia proposta sia, seppure nella sua semplicità, adatta a stimare i valori della durezza sia in campo macroscopico, che in campo microscopico, che, infine, in campo nanoscopico, e questo indipendentemente dalla dimensione assoluta dell'impronta utilizzata per la calibrazione del metodo stesso. Un tale approccio, seppure fondato su un forte pragmatismo ingegneristico, ha dato ottimi risultati, dimostrandosi, pertanto, un valido strumento da utilizzarsi in situazioni ingegneristiche di interesse pratico.

\section{CONCLUSIONI}

$\checkmark$ Le prove sperimentali condotte su tre materiali metallici diversi (Al 7075-T6, BS970-En3B e AISI 316L) hanno evidenziato un incremento della durezza rilevata al diminuire della superficie indentata;

$\checkmark$ Le simulazioni agli elementi finiti hanno dimostrato di essere attendibili fintantoché si resta nell'ambito di validità della meccanica del continuo, ovvero fintantoché le misure delle impronte hanno dimensione superiore alla dimensione media della grana cristallina;

$\checkmark$ Le proprietà meccaniche ottenute da test di trazione condotti su microprovini si sono rivelate non sufficienti ad estendere con successo l'utilizzo della meccanica del continuo alla simulazione di micro- e nano-deformazioni (come quelle di interesse in test di micro- e nano-durezza);

$\checkmark$ La metodologia ingegneristica proposta si è dimostrata un utile strumento da utilizzarsi, unitamente ai risultati numerici ottenibili con i più comuni codici di calcolo agli elementi finiti, in situazioni di interesse pratico per stimare la durezza dei materiali metallici, e questo indipendentemente dalla dimensione della superficie indentata.

\section{BIBLIOGRAFIA}

[1] A. G. Atkins, D. Tabor, Journal of Mechanics and Physics of Solids, 13 (1965) 149.

[2] K. L. Johnson, Journal of Mechanics and Physics of Solids, 18 (1970) 115.

[3] C. Rubenstein, Journal of Applied Mechanics, 48 (1981) 796.

[4] K. W. McElhaney, J. J. Vlassak, W. D. Nix, Journal of Material Research, 13 (1995) 1300.

[5] D. Tabor, The hardness of metals, Clarendon Press, Oxford, UK (1951).

[6] N. A. Stelmashenko, M. G. Wallas, L. M. Brown, Y. V. Milman, Acta Metallurgica Materialia, 41 (1993) 2855.

[7] Q. Ma, D. R. Clarke, Journal of Materials Research, 10 (1995) 853.

[8] M. F. Ashby, Philos. Mag., 21 (1970) 399.

[9] N. A. Fleck, G. M. Muller, M. F. Ashby, J. W. Hutchinson, Acta Metallurgica Materialia, 42 (1994) 475.

[10] W. D. Nix, H. Gao, Journal of Mechanics and Physics of Solids, 46 (1995) 411.

[11] B. Atzori, Appunti di Costruzione di Macchine. Ed. Libreria Cortina, Padova (2000).

[12] A. E. Giannakopoulos, P. L. Larsson, R. Vestergaard, Int. J. of Solids and Structures 31, (1994) 2679.

[13] Anon., ANSYS ${ }^{\circledR}$ Reference Guide, (2011).

[14] S. Wiersma, D. Taylor, Fatigue \& Fracture Engn. Mater. Struct., 28 (2005)1153. 